\title{
On the role of partial Ricci curvature in the geometry of submanifolds and foliations
}

\author{
by VLADIMIR ROVENSKII (Krasnoyarsk)
}

\begin{abstract}
Submanifolds and foliations with restrictions on $q$-Ricci curvature are studied. In $\S 1$ we estimate the distance between two compact submanifolds in a space of positive $q$-Ricci curvature, and give applications to special classes of submanifolds and foliations: $k$-saddle, totally geodesic, with nonpositive extrinsic $q$-Ricci curvature. In $\S 2$ we generalize a lemma by $\mathrm{T}$. Otsuki on asymptotic vectors of a bilinear form and then estimate from below the radius of an immersed submanifold in a simply connected Riemannian space with nonpositive curvature; moreover, we prove a theorem on nonembedding into a circular cylinder when the ambient space is Euclidean. Corollaries are nonembedding theorems of Riemannian manifolds with nonpositive $q$-Ricci curvature into a Euclidean space. In $\S 3$ a lower estimate of the index of relative nullity of a submanifold with nonpositive extrinsic $q$-Ricci curvature is proven. Corollaries are extremal theorems for a compact submanifold with the nullity foliation in a Riemannian space of positive curvature. On the way, some results by T. Frankel, K. Kenmotsu and C. Xia, J. Morvan, A. Borisenko, S. Tanno, B. O’Neill, J. Moore, T. Ishihara, H. Jacobowitz, L. Florit, M. Dajczer and L. Rodríguez are generalized.
\end{abstract}

The relations between curvature and topology of (sub) manifolds play an essential role in Global Riemannian Geometry. Note that the results on Ricci curvature are often based on more complicated techniques from Riemannian geometry than the results on sectional curvature.

$\mathrm{H}$. $\mathrm{Wu}[\mathrm{Wu}]$ (and later Z. Shen [Shen]) studied certain curvature functions on a Riemannian manifold $M$ which "interpolate" between the sectional curvature and the Ricci curvature. By definition, for $q+1$ orthonormal vectors $\left\{x_{0} ; x_{1}, \ldots, x_{q}\right\} \subset T M$ the (partial) q-Ricci curvature is given by the formula

$$
\operatorname{Ric}^{q}\left(x_{0} ; x_{1}, \ldots x_{q}\right)=\sum_{i=1}^{q} K\left(x_{0}, x_{i}\right)
$$

1991 Mathematics Subject Classification: 53C12, 53C40.

Key words and phrases: Riemannian manifold, submanifold, foliation, ruled submanifold, $q$-Ricci curvature, distance, radius of submanifold, index of relative nullity. 
In fact, the $q$-Ricci curvature $\operatorname{Ric}^{q}\left(x_{0} ; x_{1}, \ldots x_{q}\right)$ depends on the vector $x_{0}$ and the $q$-dimensional subspace $V=\left\{x_{1}, \ldots, x_{q}\right\}$, which is orthogonal to $x_{0}$. Obviously, the 1-Ricci curvature coincides with sectional curvature, and the ( $\operatorname{dim} M-1)$-Ricci curvature is the usual Ricci curvature. In view of the inductive identity

$$
\sum_{i=1}^{q+1} K\left(x, z_{i}\right)=\frac{1}{q} \sum_{i=1}^{q+1} \sum_{j \neq i} K\left(x, z_{j}\right),
$$

if for $M^{n}$ the inequality $\operatorname{Ric}_{M}^{q} \geq q c\left(\right.$ or $\operatorname{Ric}_{M}^{q} \leq q c$ ) holds, then $\operatorname{Ric}_{M}^{q+1} \geq$ $(q+1) c$ (resp., $\left.\operatorname{Ric}_{M}^{q+1} \leq(q+1) c\right)$. For example, since the product of two round spheres $M^{2 n}=S^{n}(1) \times S^{n}(1)$, where $n>2$, has Ricci curvature $\operatorname{Ric}_{M}=\operatorname{Ric}_{M}^{2 n-1}=n-1$ and sectional curvature $K_{M}=\operatorname{Ric}_{M}^{1} \in[0,1]$, the $(n+1)$-Ricci curvature of $M^{2 n}$ is positive: $\operatorname{Ric}_{M}^{n+1} \geq 1$. Hence, the class of Riemannian manifolds with positive $q$-Ricci curvature is larger than the class of manifolds with positive sectional curvature. Note that if for some $1<q<\operatorname{dim} M-1$ the partial curvature $\operatorname{Ric}^{q}$ is constant, then $M$ is a space of constant sectional curvature.

Let $h: \mathbb{R}^{n} \times \mathbb{R}^{n} \rightarrow \mathbb{R}^{p}$ be a symmetric bilinear map (in particular, $h$ can be the second fundamental form $h: T_{m} M \times T_{m} M \rightarrow T_{m} M^{\perp}$ at a point $m$ of a submanifold $M \subset \bar{M}$ ) and $\left\{x_{i}\right\}_{0 \leq i \leq q} \subset \mathbb{R}^{n}$ an orthonormal system of $q+1$ vectors. We define the extrinsic q-Ricci curvature of $h$ by the formula

$$
\operatorname{Ric}_{h}^{q}\left(x_{0} ; x_{1}, \ldots, x_{q}\right)=\sum_{i=1}^{q}\left[\left(h\left(x_{0}, x_{0}\right), h\left(x_{i}, x_{i}\right)\right)-h^{2}\left(x_{0}, x_{i}\right)\right],
$$

where $($,$) is the scalar product in \mathbb{R}^{p}$. For $q=1$ it is called an extrinsic sectional curvature (see [Bor 1,2], [Flo], [KN]). In view of the equality

$$
\operatorname{Ric}_{h}^{q}\left(x_{0} ; x_{1}, \ldots, x_{q}\right)=\sum_{i=1}^{q} \operatorname{Ric}_{h}^{1}\left(x_{0}, x_{i}\right),
$$

an inductive formula analogous to (1) is satisfied:

$$
\operatorname{Ric}_{h}^{q+1}\left(x_{0} ; x_{1}, \ldots, x_{q+1}\right)=\frac{1}{q} \sum_{i=1}^{q+1} \operatorname{Ric}_{h}^{q}\left(x_{0} ; x_{1}, \ldots, \widehat{x}_{i}, \ldots, x_{q+1}\right),
$$

where the symbol ${ }^{\wedge}$ over a vector means the absence of this vector. Hence for any $1 \leq q<n-1$ the inequality $\operatorname{Ric}_{h}^{q} \leq q c$ implies $\operatorname{Ric}_{h}^{q+1} \leq(q+1) c$, and $\operatorname{Ric}_{h}^{q} \equiv 0$ implies $\operatorname{Ric}_{h}^{1} \equiv 0$. For a submanifold $M \subset \bar{M}$ with second fundamental form $h$ and for any orthonormal vectors $\left\{x_{i}\right\}_{0 \leq i \leq q}$ from the tangent space $T_{m} M$ at an arbitrary point $m \in M$ the following equality holds:

(2) $\operatorname{Ric}_{M}^{q}\left(x_{0} ; x_{1}, \ldots, x_{q}\right)-\operatorname{Ric}_{\bar{M}}^{q}\left(x_{0} ; x_{1}, \ldots, x_{q}\right)=\operatorname{Ric}_{h}^{q}\left(x_{0} ; x_{1}, \ldots, x_{q}\right)$; 
in particular, for a submanifold $M$ in a Euclidean space we have

$$
\operatorname{Ric}_{M}^{q}\left(x_{0} ; x_{1}, \ldots, x_{q}\right)=\operatorname{Ric}_{h}^{q}\left(x_{0} ; x_{1}, \ldots, x_{q}\right) .
$$

In the present paper we discuss the role of $q$-Ricci curvature in the theory of submanifolds and foliations.

In $\S 1$ we estimate the distance between two compact submanifolds in a space of positive $q$-Ricci curvature, and give applications to the following types of submanifolds: $k$-saddle, totally geodesic, with nonpositive extrinsic $q$-Ricci curvature. As a corollary, we find an upper bound for the dimension of a compact $k$-saddle (in particular, totally geodesic and with nonpositive extrinsic $q$-Ricci curvature) foliation with positive mixed $q$-Ricci curvature. On the way, the results on $q$-Ricci curvature by K. Kenmotsu and C. Xia [KX 1-2], and the results on sectional curvature by A. Borisenko [1, 2], J. Morvan [Mor], S. Tanno [Tan] and B. O'Neill [O'N], relating to T. Frankel's result [Fra], are generalized.

In $\S 2$ we generalize a lemma by T. Otsuki [Ots] on asymptotic vectors of a bilinear form. As an application, we estimate from below the radius of an immersed submanifold in a simply connected Riemannian space with nonpositive curvature. A corollary is a nonembedding theorem of a compact $n$-dimensional Riemannian manifold with nonpositive $q$-Ricci curvature into a $(2 n-q)$-dimensional Euclidean space. Moreover, for the ambient Euclidean space we prove a theorem on nonembedding into a circular cylinder. On the way, the results on sectional curvature by T. Ishihara [Ish], H. Jacobowitz [Jac] and J. Moore [Moo 1] are generalized.

In $\S 3$ a lower estimate of the index of relative nullity of a submanifold with nonpositive extrinsic $q$-Ricci curvature and small codimension is given. Corollaries are extremal theorems for compact submanifolds with nullity foliations in a Riemannian space with positive curvature and also tests for totally geodesic submanifolds. On the way, the results on sectional curvature by L. Florit [Flo], A. Borisenko [Bor 2, 3], and M. Dajczer and L. Rodríguez $[\mathrm{DR}]$ are generalized.

1. The distance between two submanifolds in a space of positive $q$-Ricci curvature. By using the formula for the second variation of the length (or energy) of a geodesic, T. Frankel [Fra] showed that two compact totally geodesic submanifolds $M_{1}, M_{2}$ in a Riemannian space $M$ with positive sectional curvature (for example, two great spheres in a round sphere) must necessarily intersect if their dimensions sum to at least the dimension of $M$. If $M$ is also a Kähler manifold with positive bisectional curvature, then two compact analytic submanifolds $M_{1}, M_{2}$ must necessarily intersect if their dimensions sum to at least the dimension of $M$ (see [Fra], [GK]). These results were applied by S. Tanno [Tan], K. Abe [Abe 1] 
and A. Borisenko [Bor 1-3] to estimating the dimension of a compact totally geodesic foliation on a Riemannian manifold (or submanifold) with positive mixed sectional curvature. K. Kenmotsu and C. Xia [KX 1-2] generalized the above result of T. Frankel to the case of positive $q$-Ricci curvature.

If the radius of a circle of $S^{2}$ is "small", it is possible to find a great circle of $S^{2}$ which is "far" from it. Starting from this elementary fact J. Morvan [Mor] generalized T. Frankel's result [Fra] by giving an upper bound for the distance of two submanifolds of a Riemannian space with positive sectional curvature in terms of the lengths of their second fundamental forms.

In this section we generalize the results by J. Morvan and K. KenmotsuC. Xia to submanifolds and give applications to foliated manifolds.

Note that there are two mixed $q$-Ricci curvatures for a foliated Riemannian manifold $(M,\{L\}): \operatorname{Ric}_{1}^{q}\left(y_{0} ; x_{1}, \ldots, x_{q}\right)$ when $q \leq \operatorname{dim} L$ and $\operatorname{Ric}_{2}^{q}\left(x_{0}\right.$; $\left.y_{1}, \ldots, y_{q}\right)$ when $q \leq \operatorname{codim} L$, where $x_{i} \in T L, y_{i} \in T L^{\perp}$.

Let $\left\|h_{i}\right\|$ be the supremum of the norm of the second fundamental form of a submanifold $M_{i}$ in a Riemannian manifold $M$, and $\left|H_{i}\right|$ the mean curvature of $M_{i}$.

Lemma 1. Let $M_{1}^{\nu_{1}}$ and $M_{2}^{\nu_{1}}$ be two compact submanifolds in a Riemannian manifold $M^{n}$ with $\operatorname{Ric}_{M}^{q} \geq c>0$ for some $q \leq \min \left\{\nu_{1}, \nu_{2}\right\}$, and let

$$
q^{\prime}=\nu_{1}+\nu_{2}-n+1 .
$$

Then the distance $d\left(M_{1}, M_{2}\right)$ between $M_{1}$ and $M_{2}$ satisfies the inequality

$$
d^{2}\left(M_{1}, M_{2}\right) \leq \begin{cases}\frac{q}{c}\left\{\left\|h_{1}\right\|+\left\|h_{2}\right\|+\frac{\pi^{2}}{4}\right\} & \text { if } q^{\prime} \leq 0, \\ \frac{q}{c}\left\{\left\|h_{1}\right\|+\left\|h_{2}\right\|\right\}+\frac{q-q^{\prime}}{c} \cdot \frac{\pi^{2}}{4} & \text { if } 0<q^{\prime}<q, \\ \frac{q}{c}\left\{\left\|h_{1}\right\|+\left\|h_{2}\right\|\right\} & \text { if } q^{\prime} \geq q .\end{cases}
$$

Remark. For $q=1$ Lemma 1 was proved in [Mor], and the first inequality is the best possible: two orthogonal great circles in $S^{3}(1)$ have $\left\|h_{1}\right\|=\left\|h_{2}\right\|=0$ and distance $\pi / 2$.

We shall prove a version of this result for foliations.

Let $\left\|h_{L}\right\|$ be the supremum of the norm of the second fundamental form of leaves and $\operatorname{diam}^{\perp} L$ the maximal distance between the leaves of a foliation $\{L\}$.

THEOREM 1. Let $\left\{L^{\nu}\right\}$ be a compact foliation on a Riemannian manifold $M^{\nu+n}$ with $\operatorname{Ric}_{M}^{q} \geq c>0$ for some $q \leq \nu$. Then 


$$
\left(\operatorname{diam}^{\perp} L\right)^{2} \leq \begin{cases}\frac{2 q}{c}\left\{\left\|h_{L}\right\|+\frac{\pi^{2}}{2}\right\} & \text { if } \nu \leq n-1, \\ \frac{2 q}{c}\left\|h_{L}\right\|+\frac{q-\nu+n-1}{c} \cdot \frac{\pi^{2}}{4} & \text { if } n-1<\nu<n-1+q, \\ \frac{2 q}{c}\left\|h_{L}\right\| & \text { if } \nu \geq n-1+q .\end{cases}
$$

Remark. For the Riemannian foliation of Theorem 1 the condition on the Ricci curvature can be changed to $\operatorname{Ric}_{1}^{q}(L) \geq c>0$, because the shortest geodesic between any two leaves (see the proof) is orthogonal to such a foliation at all its points.

Proof (of Theorem 1). Consider two leaves $L_{1}, L_{2}$ with distance $l=$ $\operatorname{dist}\left(L_{1}, L_{2}\right)$, which is reached at points $m_{1} \in L_{1}, m_{2} \in L_{2}$. An argument using the first variation formula of arc-length shows that the shortest geodesic $\gamma(t)(0 \leq t \leq 1)$ with length $l$ between $m_{1}, m_{2}$ is orthogonal to $L_{1}$ and $L_{2}$.

Consider the second case: $n-1<\nu<n-1+q$. Then the parallel displacement of $T_{m_{1}} L_{1}$ along $\gamma$ will intersect $T_{m_{2}} L_{2}$ in a $q^{\prime}$-dimensional subspace $V_{2}$, where $\nu-n+1 \leq q^{\prime}<q$. We denote by $V_{1}$ the inverse image of $V_{2}$ in $T_{m_{1}} L_{1}$. Let $T_{m_{1}} L=V_{1} \oplus V_{1}^{\prime} \oplus V^{\prime \prime}$ be the orthogonal decomposition, where the parallel image of $V_{1}^{\prime}$ is uniquely projected onto $T_{m_{2}} L_{2}$ (denote its orthogonal projection in $T_{m_{2}} L_{2}$ by $V_{2}^{\prime}$ ) and the parallel image of $V^{\prime \prime}$ is orthogonal to $T_{m_{2}} L$. (Note that $V^{\prime \prime}$ is zero for small $l$.) Let vectors $e_{1}, \ldots, e_{q^{\prime}}$ form an orthonormal basis of $V_{1}$ and continue them to parallel vector fields $\bar{e}_{1}, \ldots, \bar{e}_{q^{\prime}}$ along $\gamma$. Obviously, $\bar{e}_{1}\left(m_{2}\right), \ldots, \bar{e}_{q^{\prime}}\left(m_{2}\right)$ belong to $V_{2}$. Let vectors $a_{1}, \ldots, a_{s}$ (where $s=\operatorname{dim} V_{1}^{\prime}$ ) form an orthonormal basis of $V_{1}^{\prime}$ and vectors $b_{1}, \ldots, b_{s}$ form an orthonormal basis of $V_{2}^{\prime}$, and continue them to parallel vector fields $\bar{a}_{1}, \ldots, \bar{a}_{s}$ and $\bar{b}_{1}, \ldots, \bar{b}_{s}$ along $\gamma$.

Proposition 1. Let $V_{1}, V_{2}$ be subspaces in $\mathbb{R}^{N}$ with $\operatorname{dim} V_{1}=\operatorname{dim} V_{2}$. Then there exist orthonormal bases $\left\{a_{i}\right\} \subset V_{1},\left\{b_{i}\right\} \subset V_{2}$ (which correspond to the values of extremal angles between the given subspaces) with the property $a_{i} \perp b_{j}(i \neq j)$.

Consider the field of parallel planes $\sigma_{i}(t)$ along $\gamma$, spanned by vectors $\bar{a}_{i}(t), \bar{b}_{i}(t)$. Assume that $\left\{a_{i}\right\},\left\{b_{i}\right\}$ correspond to extremal angles between $V_{1}^{\prime}$ and the parallel image of $V_{2}^{\prime}$ (see Proposition 1). Then $\sigma_{i}(t) \perp \sigma_{j}(t)$ for $i \neq j$. We take a unit vector $\widetilde{b}_{i}(t) \in \sigma_{i}(t)$ such that $\left(\bar{a}_{i}, \widetilde{b}_{i}(t)\right)=0$. It is always possible to choose $b_{i}$ and $\widetilde{b}_{i}(t)$ with $\left(\bar{a}_{i}, \bar{b}_{i}\right) \geq 0$ and $\left(\bar{b}_{i}, \widetilde{b}_{i}(t)\right) \geq 0$. We introduce unit vector fields along the geodesic $\gamma$,

$$
x_{i}(t)=\left(\cos \theta_{i} t\right) \bar{a}_{i}+\left(\sin \theta_{i} t\right) \widetilde{b}_{i}(t),
$$

where $\theta_{i}=\arccos \left(\bar{a}_{i}, \bar{b}_{i}\right) \in[0, \pi / 2]$. Note that $x_{i}(t) \perp x_{j}(t)$ when $i \neq j$. If $q^{\prime}+s<q$, then we complete the system $\left\{a_{i}\right\}$ by a certain number of 
orthogonal vectors from $V^{\prime \prime}$ and complete $\left\{b_{i}\right\}$ by a certain number of orthogonal vectors from the orthogonal complement to $V_{2} \oplus V_{2}^{\prime}$ in $T_{m_{2}} L$. For such additional pairs of vectors the fields $x_{i}(t)$ are defined in the same way, but with $\theta_{i}=\pi / 2$. Thus we may assume that $q^{\prime}+s=q$. Using the second variation of the energy $\mathcal{E}$ of $\gamma$ along $x_{i}(t)$ and $\bar{e}_{j}$ we obtain

$$
\begin{aligned}
\mathcal{E}_{x_{i}}^{\prime \prime}(0)= & \left(h_{L}\left(b_{i}, b_{i}\right), \gamma^{\prime}(1) / l\right)-\left(h_{L}\left(a_{i}, a_{i}\right), \gamma^{\prime}(0) / l\right)+\theta_{i}^{2} \\
& -l^{2} \int_{0}^{1} K\left(\gamma^{\prime}, x_{i}(t)\right) d t \geq 0, \\
\mathcal{E}_{\bar{e}_{j}}^{\prime \prime}(0)= & \left(h_{L}\left(\bar{e}_{j}, \bar{e}_{j}\right), \gamma^{\prime}(1) / l\right)-\left(h_{L}\left(e_{j}, e_{j}\right), \gamma^{\prime}(0) / l\right) \\
& -l^{2} \int_{0}^{1} K\left(\gamma^{\prime}, \bar{e}_{j}\right) d t \geq 0 .
\end{aligned}
$$

Since $s=q-q^{\prime} \leq q-\nu+n-1$ and $\sum_{i} \theta_{i}^{2} \leq \frac{\pi^{2}}{4} s$,

$$
\begin{aligned}
& \sum_{i=1}^{q^{\prime}}\left|\left(h_{L}\left(b_{i}, b_{i}\right), \gamma^{\prime}(1) / l\right)-\left(h_{L}\left(a_{i}, a_{i}\right), \gamma^{\prime}(0) / l\right)\right| \leq 2 q^{\prime}\left\|h_{L}\right\|, \\
& \sum_{j=1}^{s}\left|\left(h_{L}\left(\bar{e}_{j}, \bar{e}_{j}\right), \gamma^{\prime}(1) / l\right)-\left(h_{L}\left(e_{j}, e_{j}\right), \gamma^{\prime}(0) / l\right)\right| \leq 2 s\left\|h_{L}\right\|,
\end{aligned}
$$

and since by the given inequality for $\operatorname{Ric}_{M}^{q}$,

$$
\sum_{i=1}^{q^{\prime}} K\left(\gamma^{\prime}, x(t)\right)+\sum_{j=1}^{s} K\left(\gamma^{\prime}, \bar{e}_{j}\right) \geq c,
$$

from (3) it follows $l^{2} c \leq 2 q\left\|h_{L}\right\|+(q-\nu+n-1) \pi^{2} / 4$, which completes the proof of the second inequality of the theorem. The other cases are similar: for the first inequality the subspace $V_{1}$ is trivial, for the third we must only consider $q$ vectors from $V_{1}$.

From the third case of Theorem 1 we have the following

COROLLARY 1. Let $M^{n+\nu}$ be a Riemannian manifold with compact totally geodesic foliation $\left\{L^{\nu}\right\}$ and suppose $\operatorname{Ric}_{1}^{q}(L)$ is positive along some leaf. Then $\nu<n-1+q$.

Remark. For $q=1$ Corollary 1 was proved in [Tan] using the idea of [Fra].

The following result can be proved by a similar method to that for Lemma 1.

COROLlary 2. Let $M_{1}^{n-1}$ and $M_{2}^{l}$ be a complete hypersurface and a submanifold in a Riemannian space $\bar{M}^{n}$ with $\operatorname{Ric} \frac{q}{M} \geq c \geq 0$ for some $q \leq l$, 
and let one of $M_{1}^{n-1}, M_{2}^{l}$ be compact. Assume that $\bar{M}^{n}$ has positive q-Ricci curvature either at all points of $M_{1}^{n-1}$ or at all points of $M_{2}^{l}$. Then

$$
d^{2}\left(M_{1}, M_{2}\right) \cdot \frac{c}{q}<\left\|h_{1}\right\|+\left|H_{2}\right| .
$$

Remark. For $c=0$ in Corollary 2 we obtain the result of [KX 2] that a totally geodesic hypersurface $M_{1}^{n-1}$ (i.e., $h_{1}=0$ ) and a minimal submanifold $M_{2}$ (i.e., $\left.H_{2}=0\right)$ must intersect. The well-known particular case is that there exists no closed immersed minimal submanifold in an open hemisphere.

Next we consider classes of submanifolds and foliations with additional conditions on the second fundamental form.

Definition 1 [Shef]. A submanifold $M$ in a Riemannian space $\bar{M}$ is called a $k$-saddle submanifold if for every normal $\xi \in T M^{\perp}$ the second quadratic form $A_{\xi}$ has less than $k$ eigenvalues with the same sign, i.e., its canonical term is

$$
\begin{aligned}
a_{1} d x_{1}^{2}+\ldots+a_{j} d x_{j}^{2}-b_{1} d x_{j+1}^{2}-\ldots- & b_{i} d x_{j+i}^{2}, \\
& \max \{i, j\} \leq k-1, a_{s}, b_{l}>0 .
\end{aligned}
$$

A foliation $\{L\}$ on a Riemannian manifold $M$ is called a $k$-saddle foliation if every leaf is a $k$-saddle submanifold.

Obviously, totally geodesic submanifolds are 1-saddle.

Lemma 2. Let $M_{1}^{\nu_{1}}$ and $M_{2}^{\nu_{2}}$ be compact $k_{i}$-saddle submanifolds in a Riemannian manifold $M^{n}$ with $\operatorname{Ric}_{M}^{q} \geq c>0$ for some $q \leq \min \left\{\nu_{1}-\right.$ $\left.k_{1}, \nu_{2}-k_{2}\right\}+1$, and let $q^{\prime}=\nu_{1}+\nu_{2}-n-k_{1}-k_{2}+3$. Then

$$
d\left(M_{1}, M_{2}\right) \leq \begin{cases}\frac{\pi}{2} \sqrt{\frac{q}{c}} & \text { if } q^{\prime} \leq 0, \\ \frac{\pi}{2} \sqrt{\frac{q-q^{\prime}}{c}} & \text { if } 0<q^{\prime}<q, \\ 0, \text { i.e., } M_{1} \cap M_{2} \neq \emptyset, & \text { if } q^{\prime} \geq q .\end{cases}
$$

The third case of Lemma 2 (see [Rov]) implies the result of [BRT], which generalizes [Lemma 8, Bor 2] to $k$-saddle submanifolds with $q=1$, and the results for totally geodesic submanifolds: of [KX 1] for $k=0$, and of [Fra] for $k=0, q=1$.

Corollary 3 [BRT]. Let $M^{n}$ be a complete Riemannian manifold with nonnegative q-Ricci curvature and let $M_{1}^{\nu_{1}}$ and $M_{2}^{\nu_{2}}$ be complete immersed $k_{i}$-saddle submanifolds. Assume $M_{1}^{\nu_{1}}$ is closed and $M_{2}^{\nu_{2}}$ is compact. If $M^{n}$ has positive q-Ricci curvature either at all points of $M_{1}^{\nu_{1}}$ or at all points of $M_{2}^{\nu_{2}}$ and $\nu_{1}+\nu_{2} \geq n+q+k_{1}+k_{2}-3$, then $M_{1}^{\nu_{1}}$ and $M_{2}^{\nu_{2}}$ must intersect. 
We shall prove a version of Lemma 2 for foliations, using the following

LEMma 3 [Bor 2]. The maximal dimension of a subspace contained in the cone $\sum_{i=1}^{k} a_{i} x_{i}^{2}-\sum_{j=k+1}^{r} a_{j} x_{j}^{2}=0\left(a_{s}>0\right)$ is $\min \{k, r-k\}$.

THEOREM 2. Let $M^{n+\nu}$ be a Riemannian manifold with a compact $k$ saddle foliation $\left\{L^{\nu}\right\}$ and suppose $\operatorname{Ric}_{1}^{q}(L)$ is positive for some $q \leq \nu-k+1$. Then $\nu<q+n+2 k-3$. Moreover, if $\operatorname{Ric}_{M}^{q}$ is positive for some $q \leq \nu-k+1$, then

$\operatorname{diam}^{\perp} L \leq \begin{cases}\frac{\pi}{2} \sqrt{\frac{q}{c}} & \text { if } \nu \leq n+2 k-3, \\ \frac{\pi}{2} \sqrt{\frac{q-\nu+n+2 k-3}{c}} & \text { if } n+2 k-3<\nu<q+n+2 k-3 .\end{cases}$

Pr o o f. Consider two leaves $L_{1}, L_{2}$ with distance $l=\operatorname{dist}\left(L_{1}, L_{2}\right)$, which is reached at points $m_{1} \in L_{1}, m_{2} \in L_{2}$. The shortest geodesic $\gamma(t)(0 \leq$ $t \leq 1)$ with length $l$ between $m_{1}, m_{2}$ is orthogonal to $L_{1}$ and $L_{2}$. Since $L_{1}$ and $L_{2}$ are $k$-saddle submanifolds, by Lemma 3 the asymptotic cones for the quadratic forms of the normals $\xi_{1}=\gamma^{\prime}(0) / l, \xi_{2}=\gamma^{\prime}(1) / l$ at the end points contain subspaces $W_{1}, W_{2}$ with dimensions $\geq \nu-(k-1)$.

Next we follow the proof of Theorem 1 with $W_{i}$ in place of $T_{m_{i}} L$.

Definition 2 ([Bor 4] for $s=1$ ). A foliation $\{L\}$ on a Riemannian manifold $M$ is said to be of nonpositive (negative) extrinsic s-Ricci curvature $\operatorname{Ric}_{h}^{s}(L)$ if the $s$-Ricci curvature of its leaves is not more (less) than the $s$-Ricci curvature of the ambient space along the leaves.

The following lemma was proved in [Gla] for $s=1$.

Lemma 4. A submanifold $M^{n} \subset \bar{M}^{n+p}$ with partial extrinsic Ricci curvature $\operatorname{Ric}_{h}^{s} \leq 0$ is $k$-saddle with $k=p+s$.

Proof. By Lemma 5 (below) every subspace $V \subset T M$ with $\operatorname{dim} V>$ $p+s-1$ contains an asymptotic vector. Hence for every unit normal $\xi \in T M^{\perp}$ the second quadratic form $A_{\xi}$ has not more than $p+s-1$ nonzero eigenvalues with the same sign. From Definition 1 it follows that $M^{n}$ is a $(p+s)$-saddle submanifold.

In view of Lemma 4 and Theorem 2 we obtain the following

COROllary 4. 1. Let $M_{1}^{\nu_{i}}(i=1,2)$ be two compact submanifolds with nonpositive extrinsic $s_{i}$-Ricci curvature in a Riemannian space $M^{n}$ with $\operatorname{Ric}_{M}^{q} \geq c>0$ for some $q \leq \min \left\{2 \nu_{1}-s_{1}, 2 \nu_{2}-s_{2}\right\}-n+1$, and let 
$q^{\prime}=2\left(\nu_{1}+\nu_{2}\right)-3 n+3-\left(s_{1}+s_{2}\right)$. Then

$$
d\left(M_{1}, M_{2}\right) \leq \begin{cases}\frac{\pi}{2} \sqrt{\frac{q}{c}} & \text { if } q^{\prime} \leq 0, \\ \frac{\pi}{2} \sqrt{\frac{q-q^{\prime}}{c}} & \text { if } 0<q^{\prime}<q, \\ 0, \text { i.e., } M_{1} \cap M_{2} \neq \emptyset, & \text { if } q^{\prime} \geq q .\end{cases}
$$

2. If $\left\{L^{\nu}\right\}$ is a compact foliation on $M^{\nu+n}$ with extrinsic partial Ricci curvature (of leaves) $\operatorname{Ric}_{h}^{s}(L) \leq 0$ and partial mixed Ricci curvature $\operatorname{Ric}_{1}^{q}(L)$ $>0$ for some $q \leq \nu-s-n+1$, then $\nu<3 n+q+2 s-3$.

REmark. Analogous results hold for the Kählerian case with positive partial Ricci curvature (see [KX 1-2] for the totally geodesic case).

2. The radius of an immersed manifold and nonexistence of immersions. The well-known lemma by T. Otsuki [Ots] allows one to prove the nonexistence of an isometric embedding of a compact Riemannian manifold $M^{n}$ with nonpositive sectional curvature into the Euclidean space $\mathbb{R}^{2 n-1}$ (see $[\mathrm{KN}])$. Below we generalize this lemma.

Lemma 5. Let $h: \mathbb{R}^{n} \times \mathbb{R}^{n} \rightarrow \mathbb{R}^{p}$ be a symmetric bilinear map and suppose that for some integer $q \in[1, n-1]$ and real $c \geq 0$,

$$
\operatorname{Ric}_{h}^{q} \leq q c^{2}, \quad|h(x, x)|>c x^{2} \quad(x \neq 0) .
$$

Then $p>n-q$.

Remark. For $q=1$ Lemma 5 is the result of T. Otsuki [Ots]. From Lemma 5 it follows that in the case $\operatorname{Ric}_{h}^{q} \leq 0$ and $p \leq n-q$ the symmetric bilinear map $h$ has an asymptotic vector, i.e., there exists a nonzero $x \in \mathbb{R}^{n}$ such that $h(x, x)=0$.

Pr o of (of Lemma 5). Assume the contrary, i.e., $p \leq n-q$. The smooth function $f(x)=h^{2}(x, x)$ is positive on the unit sphere $S^{n-1}$. The minimum of $f$ is reached at some unit vector $x_{0}$. Let $F(x)=f(x)-\lambda(x, x)$. Then

(a) $\frac{1}{2} d F\left(x_{0}\right) x=2\left(h\left(x_{0}, x_{0}\right), h\left(x_{0}, x\right)\right)-\lambda\left(x_{0}, x\right)=0$,

(b) $\frac{1}{2} d^{2} F\left(x_{0}\right)(x, x)=2\left(h\left(x_{0}, x_{0}\right), h(x, x)\right)+4 h^{2}\left(x_{0}, x\right)-\lambda(x, x) \geq 0$,

where $x \in \mathbb{R}^{n}$ is an arbitrary vector. From the assumptions and (4a) for $x=x_{0}$ we obtain $\lambda>2 c^{2}$. In view of (4a) the subspace $V=\left\{x: h\left(x_{0}, x\right)\right.$ $=0\}$ is orthogonal to $x_{0}$ and, obviously, $\operatorname{dim} V \geq n-p$. Since $n-p \geq q$, we can find an orthonormal system $\left\{x_{i}\right\}_{1 \leq i \leq q} \subset V$ of $q$ vectors. From $h\left(x_{0}, x_{i}\right)=0$ and $(4 \mathrm{~b})$ it follows that $\left(h\left(x_{0}, x_{0}\right), h\left(x_{i}, x_{i}\right)\right) \geq \frac{1}{2} \lambda>c^{2}$, and 
hence $\operatorname{Ric}_{h}^{q}\left(x_{0} ; x_{1}, \ldots, x_{q}\right)=\sum_{i=1}^{q}\left(h\left(x_{0}, x_{0}\right), h\left(x_{i}, x_{i}\right)\right)>q c^{2}$, contrary to assumption.

Note that for a submanifold $M \subset \bar{M}$ with second fundamental form $h$ and an orthonormal system $\left\{x_{i}\right\}_{0 \leq i \leq q} \subset T_{m} M$ of $q+1$ vectors we have

$$
\operatorname{Ric}^{q}\left(x_{0} ; x_{1}, \ldots, x_{q}\right)-\overline{\operatorname{Ric}}^{q}\left(x_{0} ; x_{1}, \ldots, x_{q}\right)=\operatorname{Ric}_{h}^{q}\left(x_{0} ; x_{1}, \ldots, x_{q}\right) .
$$

We now consider an application of Lemma 5 to submanifolds.

J. Moore [Moo 1] proved that when $\bar{M}$ is a complete simply connected Riemannian space with sectional curvature $a \leq \bar{K} \leq b \leq 0$ and $M$ a compact Riemannian manifold with $K \leq a-b$, then $M$ has no isometric immersion in $\bar{M}$ unless $\operatorname{dim} \bar{M} \geq 2 \operatorname{dim} M$ (for $b=0$ see [O'N]). On the other hand, H. Jacobowitz [Jac] showed that an isometric immersion of an $n$-dimensional compact Riemannian manifold with sectional curvatures less than $1 / d^{2}$ into $\mathbb{R}^{2 n-1}$ can never have its image in a ball of radius $d$. T. Ishihara [Ish] proved a nonembedding theorem using the T. Otsuki Lemma, which generalized both the mentioned results. Below we generalize the result of T. Ishihara [Ish] (and hence those of J. Moore [Moo 1] and M. Jacobowitz [Jac]) to the case of partial Ricci curvature.

A positive continuous function $C(b, d)(b \leq 0, d>0)$ is defined by [Ish]

$$
C(b, d)= \begin{cases}1 / d & \text { if } b=0, \\ \sqrt{-b} \operatorname{coth}(d \sqrt{-b}) & \text { if } b<0 .\end{cases}
$$

This function is decreasing with respect to $b$ and also $d$ and obviously, $C^{2}(b, d)>-b$. Let $M$ be a compact submanifold in $\bar{M}$ with distance $\bar{d}$. For any $x \in \bar{M}$ and any $r>0$ put $B(x, r)=\{y \in \bar{M}: \bar{d}(x, y)<r\}$. Then we set

$$
r(M)=\inf \{r: M \subset B(x, r)\}=\inf \{\max \{\bar{d}(x, y): y \in M\}, x \in \bar{M}\} .
$$

There is a point $x_{0} \in \bar{M}$ such that $M \subset B\left(x_{0}, r(M)\right)$. Moreover, there is a point $y_{0} \in M$ such that $\bar{d}\left(x_{0}, y_{0}\right)=r(M)$. We will call $r(M)$ the radius of $M$ in $\bar{M}$ and $B\left(x_{0}, r(M)\right)$ a minimal ball containing $M$ [Ish]. Generally, there are several minimal balls containing $M$, for example, 2 balls (hemispheres) when $M$ is a great circle in a 2 -sphere $\bar{M}$. But there is only one minimal ball for a compact manifold immersed in a Euclidean space [Ish].

TheOREM 3. Let $\bar{M}^{n+p-q}$ be a complete simply connected Riemannian space with sectional curvature $a \leq \bar{K} \leq b \leq 0$ and $M^{n}$ a compact Riemannian manifold with diameter $<d$. Assume that at every point of $M^{n}$ there is a p-dimensional subspace in the tangent space along which the partial Ricci curvature satisfies

$$
\frac{1}{q} \operatorname{Ric}_{M}^{q} \leq a+C^{2}(b, d) .
$$

Then $M^{n}$ cannot be isometrically immersed in $\bar{M}^{n+p-q}$. 
This theorem is an immediate consequence (a compact submanifold $M \subset$ $\bar{M}$ with diameter $d$ is always contained in some ball of radius $d$ ) of the following

TheOREM 4. Let $\bar{M}^{n+p-q}$ satisfy the same conditions as in Theorem 3 and $d$ be some positive constant. Let $M^{n}$ be a compact Riemannian manifold such that at every point of $M^{n}$ there is a p-dimensional subspace in the tangent space along which the inequality (6) holds. Then no isometric immersion of $M^{n}$ into $\bar{M}^{n+p-q}$ has image in a ball of radius $<d$.

For $q=1$ Theorems 3 and 4 were proved by T. Ishihara [Ish]. Note that (6) follows from a stronger inequality

$$
\frac{1}{q} \operatorname{Ric}_{M}^{q} \leq a-b .
$$

Proof of Theorem 4. Suppose that $M^{n}$ is isometrically immersed in $\bar{M}^{n+p-q}$ and contained in a ball of radius $<d$. Then we have $r(M)<d$. Take $m_{0} \in \bar{M}$ and $m_{1} \in M$ satisfying $r(M)=\bar{d}\left(m_{0}, m_{1}\right)$. Let $\gamma:[0,1] \rightarrow \bar{M}$ be a minimal geodesic with $\gamma(0)=m_{0}, \gamma(1)=m_{1}$. For each unit tangent vector $y \in T_{m_{1}} M$ there is a unique Jacobi vector field $Y$ along $\gamma$ such that $Y(0)=0, Y(1)=y$. Corresponding to $Y$ we have a 1-parameter family of geodesics from $m_{0}$ to $M, \gamma_{s}(t)=\gamma(s, t)(|s|<\varepsilon, t \in[0,1])$, which satisfy $\gamma_{0}(t)=\gamma(t),(\partial / \partial s) \gamma(0, t)=Y(t)$. We set $\mathcal{E}\left(\gamma_{s}\right)=\frac{1}{2} \int_{0}^{1}\left(\gamma_{s}^{\prime}, \gamma_{s}^{\prime}\right) d t$, the energy of $\gamma_{s}$. Then from the definition of $\gamma$ it follows that $\mathcal{E}\left(\gamma_{s}\right) \leq \mathcal{E}(\gamma)$. Hence

$$
0 \geq\left(\frac{d^{2}}{d s^{2}} \mathcal{E}\left(\gamma_{s}\right)\right)_{s=0}=I(Y, Y)+\left(h(y, y), \gamma^{\prime}(1)\right),
$$

where $I($,$) is the index form (see [\mathrm{KN}]$ ). Taking a proper Jacobi field on a space of constant curvature $b$, J. Moore [Moo 1] proved that

$$
I(Y, Y) \geq r(M) C(b, r(M)) .
$$

Hence from (7) we get $\left(h(y, y), \gamma^{\prime}(1)\right)<-r(M) C(b, r(M))$. Since $C(b, d)$ is decreasing, it follows that $C(b, r(M))>C(b, d)$. In view of $\left|\gamma^{\prime}(1)\right|=r(M)$ we obtain for all unit vectors $y \in T_{m_{1}} M$ the inequality

$$
|h(y, y)| \geq C(b, r(M))>C(b, d) .
$$

From the conditions on the sectional curvature of $\bar{M}$ it follows that $\operatorname{Ric} \frac{q}{M} \geq$ $q a$. Hence, from (6), in view of (5), the restriction $h_{1}: V \times V \rightarrow T_{m_{1}} M^{\perp}$ of the second fundamental form of $M^{n}$ to some $p$-dimensional subspace $V \subset T_{m_{1}} M$ satisfies the inequality

$$
\operatorname{Ric}_{h_{1}}^{q} \leq q C^{2}(b, d) .
$$

But then Lemma 5 yields a contradiction: $p-q>\operatorname{dim} V-q=p-q$. 
COROllary 5. (a) Let $M^{n}$ be a compact Riemannian manifold with $\operatorname{Ric}_{M}^{q} \leq q c^{2}$ for some integer $q \in[1, n-1]$ and a constant $c>0$. Then $M^{n}$ cannot be isometrically immersed in $\mathbb{R}^{2 n-q}$ into a ball of radius $r=1 / c$.

(b) Let $M^{n}$ be a compact Riemannian manifold with nonpositive $q$-Ricci curvature. Then $M^{n}$ cannot be isometrically immersed into $\mathbb{R}^{2 n-q}$.

(c) Let $M^{n}$ be a Riemannian manifold with negative q-Ricci curvature. Then $M^{n}$ cannot be isometrically immersed into $\mathbb{R}^{2 n-q-1}$.

For $q=1$ Corollary 5 was proved by S. Chern and N. Kuiper (see $[\mathrm{KN}]$ ).

Pr o of (of Corollary 5). (a) directly follows from Theorem 4.

(b) Assume the contrary, i.e., $M^{n} \subset \mathbb{R}^{n+p}$, where $p \leq n-q$. By Lemma 5 (with $c=0$ ) for each $m \in M$ there exists an asymptotic vector $x \in T_{m} M$. But for a compact submanifold $M^{n} \subset \mathbb{R}^{n+p}$ there exist a point $m_{0} \in M$ and a unit normal $\xi_{0} \in T_{m_{0}} M^{\perp}$ with positive second quadratic form $A_{\xi_{0}}$ (see $[\mathrm{KN}]$ ). Hence, there are no asymptotic vectors at $m_{0}$, which is a contradiction.

(c) Assume the contrary, i.e., $M^{n} \subset \mathbb{R}^{n+p}$, where $p<n-q$. By Lemma 5 (with $c=0$ ) for any $m \in M$ there exists a unit asymptotic vector $x_{0} \in$ $T_{m} M$. Note that the subspace $V=\left\{x \in T_{m} M: h\left(x_{0}, x\right)=\left(x_{0}, x\right)=0\right\}$ has $\operatorname{dim} V \geq q$. Then for any orthonormal system $\left\{x_{i}\right\}_{1 \leq i \leq q} \subset V$ we have $\operatorname{Ric}^{q}\left(x_{0} ; x_{1}, \ldots, x_{q}\right)=\operatorname{Ric}_{h}^{q}\left(x_{0} ; x_{1}, \ldots, x_{q}\right)=0$, which is a contradiction.

Note that the product $M^{2 n}=M^{n}(-1) \times M^{n}(-1)$ of hyperbolic space forms has sectional curvature $K_{M} \in[-1,0]$, constant Ricci curvature $\operatorname{Ric}_{M}$ $=-(n-1)$ and hence $\operatorname{Ric}_{M}^{n+1} \leq-1$ and $\operatorname{Ric}_{M}^{n} \leq 0$. By Corollary 5 such an $M^{2 n}$ cannot be isometrically locally immersed into $\mathbb{R}^{3 n-2}$.

Corollary 5 can be improved in the case of embeddings into a cylinder of a Euclidean space.

Definition 3. A hypersurface $C(s, r)$ in $\mathbb{R}^{N+1}$ which is congruent to

$$
\sum_{i=1}^{s+1}\left(x_{i}\right)^{2}-r^{2}=0
$$

is called a circular cylinder of radius $r$ with $s$-dimensional parallel and $(N-s)$-dimensional ruling (generator, or axes).

For $s=N$ we obtain a hypersphere of radius $r>0$.

TheOREM 5. Let $M^{n}$ be a compact manifold with $\operatorname{Ric}_{M}^{q} \leq q c^{2}$ for some integer $q \in[1, n-1]$ and a constant $c>0$. Then $M^{n}$ cannot be isometrically embedded into $\mathbb{R}^{n+p}(p \leq n-q)$ inside a circular cylinder of radius $r=1 / c$ with $(2 p+q-1)$-dimensional parallel.

Proof. Suppose the contrary, i.e., $M^{n}$ is isometrically embedded into $\mathbb{R}^{n+p}(p \leq n-q)$ inside a circular cylinder $C(2 p+q-1, r)$ of radius $r=1 / c$ 
with $(2 p+q-1)$-dimensional parallel. Since the dimension $n$ of the manifold is greater than the dimension $n-p-q$ of the axes of the cylinder $C(2 p+q-1, r)$, decreasing the radius $r$ and keeping the axes we obtain a smaller cylinder $C\left(2 p+q-1, r_{1}\right)$ of radius $r_{1} \in(0, r)$ which is tangent to $M^{n}$ at some point $m$.

Consider the field of unit normals $\overline{\mathbf{n}}$ to the cylinder $C\left(2 p+q-1, r_{1}\right)$, pointing inwards. Then the principal curvatures $\left\{k_{i}(\overline{\mathbf{n}})\right\}$ of $C\left(2 p+q-1, r_{1}\right)$ are nonnegative, and

$$
0=k_{1}(\overline{\mathbf{n}})=\ldots=k_{n-p-q}(\overline{\mathbf{n}})<k_{n-p-q+1}(\overline{\mathbf{n}})=\ldots=k_{n+p-1}(\overline{\mathbf{n}})=1 / r_{1} .
$$

In view of the relation between the dimensions of the cylinder, its parallel and the submanifold $M^{n}: n+(2 p+q-1)-(n+p-1)=p+q$, there exists a $(p+q)$-dimensional subspace $T_{1} \subset T_{m} M$ which is tangent to a parallel of cylinder.

The principal curvatures of $C\left(2 p+q-1, r_{1}\right)$ in directions $x \in T_{1}$ are constant and equal to the principal curvature $1 / r_{1}$ of a parallel $((2 p+q-1)$ dimensional sphere) $S\left(r_{1}\right)$. Since the principal curvature $A_{\overline{\mathbf{n}}(m)}(x, x)$ of $M^{n}$ for the normal $\overline{\mathbf{n}}(m)$ in direction $x \in T_{1}$ is not less than $1 / r_{1}$, in view of the formula

$$
A_{\overline{\mathbf{n}}(m)}(x, x)=(h(x, x), \overline{\mathbf{n}}(m)),
$$

we have the following estimate for the second fundamental form $h$ of $M^{n}$ in directions of $T_{1}$ :

$$
|h(x, x)| \stackrel{(8)}{\geq} A_{\overline{\mathbf{n}}(m)}(x, x)>\frac{1}{r} x^{2} \quad\left(x \in T_{1}\right) .
$$

Hence there exist $q+1$ orthonormal vectors $\left\{x_{0} ; x_{1}, \ldots, x_{q}\right\}$ in $T_{1}$ with

$$
\operatorname{Ric}_{M}^{q}\left(x_{0} ; x_{1}, \ldots, x_{q}\right) \stackrel{\left(2^{\prime}\right)}{=} \operatorname{Ric}_{h}^{q}\left(x_{0} ; x_{1}, \ldots, x_{q}\right)>\frac{q}{r^{2}},
$$

because in the opposite case, by Lemma 5 for the restriction of the second fundamental form $h: T_{1} \times T_{1} \rightarrow T_{m} M^{\perp}$, we obtain $p>\operatorname{dim} T_{1}-q=p$, a contradiction.

3. The index of relative nullity and extremal theorems for $q$ Ricci curvature. We consider a symmetric bilinear form $h$ with nonpositive extrinsic $q$-Ricci curvature more carefully and obtain the best estimate of its index of relative nullity.

Lemma 6. Let $h: \mathbb{R}^{n} \times \mathbb{R}^{n} \rightarrow \mathbb{R}^{p}$ be a symmetric bilinear map with $\operatorname{Ric}_{h}^{q} \leq 0$. Then $\mu(h) \geq n-2 p-q+\delta_{1 q}$.

For $q=1$ Lemma 6 was proved by L. Florit [Flo]. Note that from $\operatorname{Ric}_{h}^{q}$ $\equiv 0$, where $q<n-1$, it follows that $\operatorname{Ric}_{h}^{1} \equiv 0$ and then (see $[\mathrm{KN}]$ ) $\mu(h) \geq n-p$. From Lemma 6 we obtain an estimate for the index $\mu(M)$ 
of relative nullity of a submanifold $M^{n} \subset \bar{M}^{n+p}$ with nonpositive extrinsic $q$-Ricci curvature and "small" codimension (for $q=1$ see [Flo] and [Bor 1]):

$$
\mu(M) \geq n-2 p-q+\delta_{1 q} .
$$

Lemma 6 follows immediately from Lemmas 7, 8 given below.

Definition 4 [Moo 2]. We say that $y \in \mathbb{R}^{n}$ is a regular element of a bilinear map $\beta: \mathbb{R}^{n} \times \mathbb{R}^{m} \rightarrow \mathbb{R}^{p}$ if $\operatorname{dim} \operatorname{Im} \beta(y)=\max \left\{\operatorname{dim} \operatorname{Im} \beta(z): z \in \mathbb{R}^{n}\right\}$. The set of regular elements of $\beta$ is denoted by $\operatorname{RE}(\beta)$.

Note that the set $\operatorname{RE}(\beta)$ is open and dense in $\mathbb{R}^{p}$.

Lemma 7 [Moo 2]. Let $\beta: \mathbb{R}^{n} \times \mathbb{R}^{m} \rightarrow \mathbb{R}^{p}$ be a bilinear map and $y_{0} \in$ $\operatorname{RE}(\beta)$. Then $\beta\left(y, \operatorname{ker} \beta\left(y_{0}\right)\right) \subset \operatorname{Im} \beta\left(y_{0}\right)$ for all $y \in \mathbb{R}^{n}$.

Proof. Let $z_{1}, \ldots, z_{r}$ be vectors in $\mathbb{R}^{m}$ with $r=\operatorname{dim} \operatorname{Im} \beta\left(y_{0}\right)$ and $\operatorname{Im} \beta\left(y_{0}\right)=\operatorname{span}\left\{\beta\left(y_{0}, z_{j}\right): 1 \leq j \leq r\right\}$. It is easy to see that the vectors $\left\{\beta\left(y_{0}+t y, z_{j}\right)\right\}_{1 \leq j \leq r}$ are linearly independent except for a finite number of values of $t$. Hence, they generate a family of $r$-dimensional subspaces that varies continuously with $t$ if $|t|<\varepsilon$ for some $\varepsilon>0$. But if $z \in \operatorname{ker} \beta\left(y_{0}\right)$, then $\beta\left(y_{0}+t y, z\right)=t \beta(y, z)$. Therefore, by continuity, $\beta(y, z) \in \operatorname{Im} \beta\left(y_{0}\right)$.

We denote the set of asymptotic vectors of $h$ by As $(h)$. Recall that $T \subset$ $\mathbb{R}^{n}$ is an asymptotic subspace of $h$ if $h(x, y)=0$ for all $x, y \in T$.

The following result, which for $q=1$ was proved in [Flo], generalizes Lemma 5.

LEMma 8. Let $h: \mathbb{R}^{n} \times \mathbb{R}^{n} \rightarrow \mathbb{R}^{p}$ be a symmetric bilinear map with $\operatorname{Ric}_{h}^{q} \leq 0$. Then there exists an asymptotic subspace $T \subset \mathbb{R}^{n}$ of $h$ such that $\operatorname{dim} T \geq n-p-q+\delta_{1 q}$.

Proof. If $n-p-q+\delta_{1 q}=1$, then by Lemma 5 there exists an asymptotic vector and this concludes the proof. Thus assume $n-p-q+\delta_{1 q} \geq 2$. For each vector $x_{0} \in \operatorname{As}(h)$ which is a regular element for $h$, we define a linear transformation $h\left(x_{0}\right): \mathbb{R}^{n} \rightarrow \mathbb{R}^{p}$ by $h\left(x_{0}\right) y=h\left(x_{0}, y\right)$, set $V_{1}=V_{1}\left(x_{0}\right)=$ ker $h\left(x_{0}\right), W_{1}=W_{1}\left(x_{0}\right)=\left\{\operatorname{Im} h\left(x_{0}\right)\right\}^{\perp}$, and define $h_{1}=h_{\mid V_{1} \times V_{1}}$. With the above notations we claim that $\operatorname{Im} h_{1} \subset W_{1}$.

From the above assumption, $\operatorname{dim} V_{1} \geq n-p \geq q+2-\delta_{1 q}$. To prove the claim, take an orthonormal system $\left\{z_{i}\right\}_{\delta_{1 q} \leq i \leq q} \subset V_{1}$ of vectors which are orthogonal to $x_{0}$. Then, since $x_{0} \in \operatorname{As}(h)$, we have for any unit $y \in \mathbb{R}^{n}$ and for all $t$,

$$
\begin{aligned}
& \left(h\left(x_{0}+t y, x_{0}+t y\right), h\left(z_{i}, z_{i}\right)\right)-\left(h\left(x_{0}+t y, z_{i}\right), h\left(x_{0}+t y, z_{i}\right)\right) \\
& \quad=2 t\left(h\left(x_{0}, y\right), h\left(z_{i}, z_{i}\right)\right)+t^{2}\left[\left(h(y, y), h\left(z_{i}, z_{i}\right)\right)-\left(h\left(y, z_{i}\right), h\left(y, z_{i}\right)\right)\right] .
\end{aligned}
$$

We can assume $y \perp V_{1}$ (see the coefficient of $t$ ), and then the unit vector 
$x_{t}=\left(1+t^{2}\right)^{-1 / 2}\left(x_{0}+t y\right)$ is orthogonal to each $z_{i}$. Hence for all $t$,

$$
\begin{aligned}
\left(1+t^{2}\right) \operatorname{Ric}_{h}^{q}\left(x_{t} ; z_{1}, \ldots, z_{q}\right)= & 2 t\left(h\left(x_{0}, y\right), \sum_{i=1}^{q} h\left(z_{i}, z_{i}\right)\right) \\
& +t^{2} \operatorname{Ric}_{h}^{q}\left(y ; z_{1}, \ldots, z_{q}\right) .
\end{aligned}
$$

In view of $\operatorname{Ric}_{h}^{q} \leq 0$, we have $\left(h\left(x_{0}, y\right), \sum_{i=1}^{q} h\left(z_{i}, z_{i}\right)\right)=0\left(y \in \mathbb{R}^{n}\right)$, i.e., $\sum_{i=1}^{q} h\left(z_{i}, z_{i}\right) \subset W_{1}$. Note that for $q=1$ we have $h(z, z) \subset W_{1}$ for all $z \in V_{1}$ and by symmetry of $h$ the claim is proved.

So assume $q>1$. Since the analogous property $\sum_{i=0}^{q-1} h\left(z_{i}, z_{i}\right) \subset W_{1}$ holds, we have $h\left(z_{0}, z_{0}\right)-h\left(z_{q}, z_{q}\right) \subset W_{1}$. In the same way we obtain $h\left(z_{0}, z_{0}\right)-h\left(z_{i}, z_{i}\right) \subset W_{1}$ for each $i$ and hence

$$
h\left(z_{0}, z_{0}\right)=\frac{1}{q} \sum_{i=1}^{q}\left[h\left(z_{0}, z_{0}\right)-h\left(z_{i}, z_{i}\right)\right]+\frac{1}{q} \sum_{i=1}^{q} h\left(z_{i}, z_{i}\right) \subset W_{1} .
$$

Since $z_{0}$ is an arbitrary unit vector in $V_{1}$, by symmetry of $h$ we have $\operatorname{Im} h_{1} \subset$ $W_{1}$.

The above claim allows us to proceed inductively as follows. Set $V_{0}=\mathbb{R}^{n}$ and $W_{0}=\mathbb{R}^{p}$. Given $k \geq 0$, for the symmetric bilinear map $h_{k}=h_{\mid V_{k} \times V_{k}}$ : $V_{k} \times V_{k} \rightarrow W_{k}$ with nonpositive $\operatorname{Ric}_{h_{k}}^{q}$, define

$$
r_{k}=\max \left\{\operatorname{dim} \operatorname{Im} h_{k}(x): x \in \operatorname{As}\left(h_{k}\right)\right\},
$$

and suppose that if $k \geq 1$, then

$$
n_{k}=\operatorname{dim} V_{k}=n-\sum_{i=0}^{k-1} r_{i}, \quad p_{k}=\operatorname{dim} W_{k}=p-\sum_{i=0}^{k-1} r_{i} .
$$

Picking $x_{k} \in \operatorname{As}\left(h_{k}\right)$ with $\operatorname{dim} \operatorname{Im} h_{k}\left(x_{k}\right)=r_{k}$, set $V_{k+1}=V_{k+1}\left(x_{0}, \ldots, x_{k}\right)$ $=\operatorname{ker} h_{k}\left(x_{k}\right) \subset W_{k}$, and then $n_{k+1}=\operatorname{dim} V_{k+1}=n-\sum_{i=0}^{k} r_{i}$. The above claim implies that $\operatorname{Im} h_{k+1} \subset W_{k+1}$, where $W_{k+1}=W_{k+1}\left(x_{0}, \ldots, x_{k}\right)=$ $\left\{\operatorname{Im} h_{k}\left(x_{k}\right)\right\}^{\perp} \subset W_{k}$, and $h_{k+1}=h_{\mid V_{k+1} \times V_{k+1}}$. Since

$$
0 \leq p_{k+1}=\operatorname{dim} W_{k+1}=p-\sum_{i=0}^{k} r_{i}
$$

there exists a positive integer $m$ such that $r_{m}=0$. This tells us that $\operatorname{As}\left(h_{m}\right)=\operatorname{ker} h_{m}$. Set $T=\operatorname{ker} h_{m}$. By Lemma 5 (with $c=0$ ) for each subspace $S \subset V_{m}$ such that $\operatorname{dim} S \geq p_{m}+q-\delta_{1 q}$, we have $S \cap T=S \cap \operatorname{As}\left(h_{m}\right)$ $\neq 0$. Hence, $\operatorname{dim} T \geq n_{m}-p_{m}+q-\delta_{1 q}=n-p+q-\delta_{1 q}$. Moreover, since $h_{m}=h_{\mid V_{m} \times V_{m}}, T$ is an asymptotic subspace of $h$ and this concludes the proof.

Proof of Lemma 6. Let $T \subset \mathbb{R}^{n}$ be an asymptotic subspace of $h$ such that $\operatorname{dim} T=n-p-q+\delta_{1 q}$ (see Lemma 8 below). Then the orthogonal 
complement $T^{\prime}$ to $T$ in $\mathbb{R}^{n}$ has $\operatorname{dim} T^{\prime}=p+q-\delta_{1 q} \geq q+1-\delta_{1 q}$. Define a bilinear map $\beta: T^{\prime} \times T \rightarrow \mathbb{R}^{p}$ by $\beta=h_{\mid T^{\prime} \times T}$. Take $y_{0} \in \operatorname{RE}(\beta), z \in T, z^{\prime} \in$ $\operatorname{ker} \beta\left(y_{0}\right) \subset T$ and an orthonormal system $\left\{y_{i}\right\}_{1 \leq i \leq q} \subset T^{\prime}$ of vectors which in case $q>1$ are also orthogonal to $y_{0}$. Using only the assumption on $T$, we have for all $s, t \in \mathbb{R}$,

$$
\begin{aligned}
& \left(h\left(y_{0}+t z, y_{0}+t z\right), h\left(y_{i}+s z^{\prime}, y_{i}+s z^{\prime}\right)\right)-h^{2}\left(y_{0}+t z, y_{i}+s z^{\prime}\right) \\
& \quad=\left(h\left(y_{0}, y_{0}\right)+2 t h\left(y_{0}, z\right), h\left(y_{i}, y_{i}\right)+2 s h\left(y_{i}, z^{\prime}\right)\right)-h^{2}\left(y_{0}+t z, y_{i}+s z^{\prime}\right) .
\end{aligned}
$$

Since $h\left(y_{0}, z^{\prime}\right)=0$, we get

$$
\begin{aligned}
&\left(h\left(y_{0}+t z, y_{0}+t z\right), h\left(y_{i}+s z^{\prime}, y_{i}+s z^{\prime}\right)\right)-h^{2}\left(y_{0}+t z, y_{i}+s z^{\prime}\right) \\
&=\left\{\left(h\left(y_{0}, y_{0}\right), h\left(y_{i}, y_{i}\right)\right)-h^{2}\left(y_{0}, y_{i}\right)\right\}-t^{2} h^{2}\left(z, y_{i}\right) \\
&\left.+2 t\left\{h\left(y_{0}, z\right), h\left(y_{i}, y_{i}\right)\right)-\left(h\left(y_{0}, y_{i}\right), h\left(z, y_{i}\right)\right)\right\} \\
&\left.+2 s\left\{h\left(y_{0}, y_{0}\right), h\left(y_{i}, z^{\prime}\right)\right)+2 t\left(h\left(y_{0}, z\right), h\left(y_{i}, z^{\prime}\right)\right)\right\},
\end{aligned}
$$

which is linear in $s$. For the unit vectors

we then obtain

$$
y(t)=\frac{1}{\sqrt{1+t^{2}}}\left(y_{0}+t z\right), \quad y_{i}(s)=\frac{1}{\sqrt{1+s^{2}}}\left(y_{i}+s z^{\prime}\right)
$$

$$
\begin{aligned}
\left(1+t^{2}\right)(1 & \left.+s^{2}\right) \operatorname{Ric}_{h}^{q}\left(y(t) ; y_{1}(s), \ldots, y_{q}(s)\right) \\
= & \operatorname{Ric}_{h}^{q}\left(y_{0} ; y_{1}, \ldots, y_{q}\right)-t^{2} \sum_{i=1}^{q} h^{2}\left(z, y_{i}\right) \\
& +2 t\left\{\left(h\left(y_{0}, z\right), \sum_{i=1}^{q} h\left(y_{i}, y_{i}\right)\right)-\sum_{i=1}^{q}\left(h\left(y_{0}, y_{i}\right), h\left(z, y_{i}\right)\right)\right\} \\
& +2 s\left\{\left(h\left(y_{0}, y_{0}\right), h\left(\sum_{i=1}^{q} y_{i}, z^{\prime}\right)\right)+2 t\left(h\left(y_{0}, z\right), h\left(\sum_{i=1}^{q} y_{i}, z^{\prime}\right)\right)\right\},
\end{aligned}
$$

which is linear in $s$. This implies, in view of $\operatorname{Ric}_{h}^{q} \leq 0$, that for all $t \in \mathbb{R}$,

$$
\left(h\left(y_{0}, y_{0}\right), h\left(\sum_{i=1}^{q} y_{i}, z^{\prime}\right)\right)+2 t\left(h\left(y_{0}, z\right), h\left(\sum_{i=1}^{q} y_{i}, z^{\prime}\right)\right)=0
$$

which says that $\left(h\left(y_{0}, y_{0}\right), h\left(\sum_{i=1}^{q} y_{i}, z^{\prime}\right)\right)=0$, and hence $\left(h\left(y_{0}, y_{0}\right), h\left(y, z^{\prime}\right)\right)$ $=0$ for all $y \in T^{\prime}$. From the arbitrariness of $z, z^{\prime}$ it follows that

$$
\beta\left(y, \operatorname{ker} \beta\left(y_{0}\right)\right) \perp \operatorname{Im} \beta\left(y_{0}\right) .
$$

This together with Lemma 7 tells us that $h(y, x)=0$ for all $y \in T^{\prime}$ and $x \in \operatorname{ker} \beta\left(y_{0}\right)$. But since $\operatorname{ker} \beta\left(y_{0}\right) \subset T$, we obtain $\operatorname{ker} \beta\left(y_{0}\right) \subset \operatorname{ker} h$. Then

$$
\mu(h) \geq \operatorname{dim} \operatorname{ker} \beta\left(y_{0}\right)=\operatorname{dim} T-\operatorname{dim} \operatorname{Im} \beta\left(y_{0}\right) \geq n-2 p-q+\delta_{1 q},
$$

which concludes the proof. 
M. Dajczer and L. Rodríguez $[\mathrm{DR}]$ have shown that any isometric immersion of a Kähler manifold into a complex space form $\bar{M}^{N}(c)(c \neq 0)$ with positive index of relative nullity must be holomorphic. The proof of that result and Lemma 6 yield the following statement, which for $q=1$ was given in [Flo].

Corollary 6. Let $M^{2 n}$ be a Kähler manifold and $m_{0} \in M$ such that $\operatorname{Ric}^{q}\left(m_{0}\right) \leq q c$, where $c$ is a positive constant. Then there is no isometric immersion of $M^{2 n}$ into a real space form $\bar{M}^{2 n+p}(c)$ for $p<n-q+\delta_{1 q}$.

P r o of. Suppose that such an immersion exists and call it $f$. Composing $f$ with the totally geodesic and totally real inclusion $i$ of the $(2 n+p)$ dimensional real space form into a certain complex space form $\widetilde{C M}^{2 n+p}(c)$, we conclude from Lemma 6 that the index of relative nullity $\mu(i \circ f)_{m_{0}}>0$. But the proof of the main result in $[\mathrm{DR}]$ shows that $T_{m_{0}} M$ must be invariant with respect to the complex structure $J$ of $M$. This is a contradiction, because $i \circ f$ is totally real.

Let $\varrho(m-1)$ be the well-known number of continuous pointwise linearly independent vector fields on the $(m-1)$-dimensional sphere. Then $\varrho\left((\right.$ odd $\left.) 2^{4 b+c}\right)=8 b+2^{c}(b \geq 0,0 \leq c \leq 3)$ and $\varrho(n) \leq 2 \log _{2} n+2$, $\varrho(n) \leq n$.

The integer sequence $\nu(n)=\max \{t: t<\varrho(n-t)\}$ was introduced in $[$ Fer].

D. Ferus [Fer] proved that any isometric immersion of a complete Riemannian manifold $M^{n}$ into a round sphere $S^{N}$ with index of relative nullity $\mu(M) \geq \varrho(n-\mu(M))$ (i.e., with $\mu(M) \geq \nu(n))$ must be totally geodesic. From [Abe 2] and [DR] it follows that any isometric immersion of a complete Kähler manifold into $\mathbb{C} P^{N}$ with positive index of relative nullity must be totally geodesic. The above results and Lemma 6 yield the following statement, which was given in [Fer] and [Flo 2] for $q=1$.

COROLlary 7. (a) Let $f: M^{n} \rightarrow S^{n+p}$ be an isometric immersion of a complete Riemannian manifold with $\operatorname{Ric}_{h}^{q} \leq 0$. If $2 p<n-\nu(n)-q+\delta_{1 q}$, then $f$ is a totally geodesic inclusion.

(b) Let $f: M^{2 n} \rightarrow \mathbb{C} P^{n+p}$ be an isometric immersion of a complete Kähler manifold with $\operatorname{Ric}_{h}^{q} \leq 0$. If $2 p<n-q+1$ then $f$ is a totally geodesic inclusion.

Next we give some extremal theorems (tests for totally geodesic submanifolds in the unit sphere) involving partial Ricci curvature, which generalize the results on sectional curvature by [Bor 3], for the first time obtained in [Bor 2] for submanifolds in CROSS (i.e., compact rank one symmetric spaces). 
A curvature invariant submanifold $M \subset \bar{M}$, i.e., one satisfying

$$
\bar{R}(x, y, z)^{\perp}=0 \quad(x, y, z \in T M),
$$

which holds, for instance, in space forms, in case $\mu(M)>0$ has the structure of a ruled developable submanifold with $\mu(M)$-dimensional generator, and intrinsically is a totally geodesic foliated manifold [Mal].

THEOREM 6. Let $M^{n} \subset \bar{M}^{n+p}$ be a complete curvature invariant submanifold with $\mathrm{Ric}_{h}^{q} \leq 0$. Then $M$ is a totally geodesic submanifold if any of the following conditions is satisfied:

1. $M$ is compact with $\operatorname{Ric}^{s}(M)>0$, and $4 p \leq n-s-2 q+2 \delta_{1 q}$,

2. $\bar{M}$ is compact with $\operatorname{Ric}^{s}(\bar{M})_{\mid M}>0$, and $5 p \leq n-s-2 q+2 \delta_{1 q}$,

3. $2 p<n-\nu(n)-q+\delta_{1 q}$ and for some $k=$ const $>0$,

$$
\bar{R}(x, y) x=-k y(x, x) \quad(x, y \in T M) .
$$

REMARK. For a submanifold $M^{n}$ in the sphere $S^{n+p}(k)$ for case 3 with $q=1$ see [Flo], for a submanifold with a stronger condition than (9) and with $q=s=1$ see [Bor 3].

Proof (of Theorem 6). The relative nullity (totally geodesic) foliation on the regularity domain has complete leaves and dimension $\mu(M) \geq n-$ $2 p-q+\delta_{1 q}$ (see Lemma 6).

1. Suppose that $M^{n}$ is not a totally geodesic submanifold. Then from $\operatorname{Ric}^{s}(M)>0$ it follows that $\mu(M)<n-\mu(M)+s$ (see Lemma 1 or Corollary 1 for the relative nullity foliation). Hence $4 p>n-s-2 q+2 \delta_{1 q}$.

2. Analogously to case 1 , from $\operatorname{Ric}^{s}(\bar{M})_{\mid M}>0$ it follows that $\mu(M)<$ $n+p-\mu(M)+s$ (see Corollary 1 ). Hence $5 p>n-s-2 q+2 \delta_{1 q}$, or $M^{n}$ is a totally geodesic submanifold.

3. Denote by $K_{\text {mix }}$ the sectional curvature in mixed directions (a plane which contains a vector tangent to the foliation and a vector orthogonal to it is said to be mixed). From $K_{\text {mix }}=k$ we have $\mu(M) \leq \nu(n)$ [Fer]. Hence $2 p \geq n-\nu(n)-q+\delta_{1 q}$, or $M$ is a totally geodesic submanifold.

Theorem 6 directly implies

TheOREM 7. Let $M^{n} \subset \bar{M}^{n+p}$ be a compact simply connected curvature invariant submanifold, and suppose one of following properties holds:

1. $\operatorname{Ric}^{s}(M) \leq s \leq \operatorname{Ric}^{s}(\bar{M})_{\mid M}$ for some $s<n-1$, and $2 p<n-\nu(n)-$ $s+\delta_{1 s}-1$,

2. $K(\bar{M})_{\mid M} \equiv 1, K_{M} \leq 1, \operatorname{inj}(M) \geq \pi$, and $2 p<n-1$.

Then $M^{n}$ is a totally geodesic submanifold which is isometric to the unit sphere. 
The property $\operatorname{inj}(M) \geq \pi$ in case 2 follows from $K_{M}>0$ when $n$ is even, and from $K_{M} \geq 1 / 4$ when $n$ is odd (see $[\mathrm{KN}]$ ).

Proof (of Theorem 7). 1. Since the extrinsic partial Ricci curvature $\operatorname{Ric}_{h}^{s} \leq 0$, by Lemma 6 we have $\mu(M)>\nu(n)+1 \geq 2$. For any orthonormal vectors $\left\{x_{0}, \ldots, x_{s}\right\} \subset T M$ with $x_{0} \in$ ker $h$ we have $\operatorname{Ric}^{s}\left(x_{0} ; x_{1}, \ldots, x_{s}\right)=$ $s=\overline{\operatorname{Ric}}^{s}\left(x_{0} ; x_{1}, \ldots, x_{s}\right)$ and hence the relative nullity foliation $\{L\}$ on $M^{n}$ has $K_{L}=K_{\text {mix }}=1$. Then from Theorem 6 , case 3 (see the proof) it follows that $\mu(M)=n$, i.e., $M^{n}$ is a totally geodesic submanifold. Since the sectional curvature $K_{M}=1$, the simply connected $M^{n}$ is isometric to the unit $n$-sphere.

2. Since the extrinsic sectional curvature is nonpositive, from Lemma 6 (with $q=1$ ) it follows that $\mu(M) \geq 2$, and hence $M^{n}$ contains a $\mu(M)$ dimensional totally geodesic submanifold $L$ (a generator) with $K_{L}=1$, which is simply connected by the condition $\operatorname{inj}(M) \geq \pi$. Since $L$ contains a closed geodesic $\gamma$ of $M^{n}$ of length $2 \pi$ and index $n-1, M^{n}$ is isometric to the unit sphere due to Toponogov's extremal theorem [Top]. Since the extrinsic sectional curvature of $M^{n}$ is zero, we have $\mu(M) \geq n-p$ (see [KN]). From the above it follows that $\mu(M) \geq n / 2+1$ and by Lemma 1 (for totally geodesic submanifolds in $\bar{M}$ with $q=1) \mu(M)=n$, i.e., $M^{n}$ is a totally geodesic submanifold.

If the curvature of a submanifold of $\bar{M}^{n+p}$ satisfies stronger restrictions than in condition 1 of Theorem 7, we obtain the following extremal theorem.

THEOREM 8. Let $M^{n}$ be a compact curvature invariant submanifold in a complete simply connected Riemannian space $\bar{M}^{n+p}$ satisfying $9 / 4 \geq K_{\bar{M}}$ $\geq 1, \operatorname{Ric}^{s}(M) \leq s$ for some $s<n-1$ and $2 p \leq n-s-2+\delta_{1 s}$. Then $\bar{M}^{n+p}$ is isometric to the unit sphere.

P r o of. Since the extrinsic curvature $\operatorname{Ric}_{h}^{s}$ is nonpositive, from Lemma 6 it follows that $\mu(M) \geq 2$. For any orthonormal vectors $\left\{x_{0}, \ldots, x_{s}\right\} \subset T M$ with $x_{0} \in \operatorname{ker} h$ we have $\operatorname{Ric}^{s}\left(x_{0} ; x_{1}, \ldots, x_{s}\right)=s=\overline{\operatorname{Ric}}^{s}\left(x_{0} ; x_{1}, \ldots, x_{s}\right)$ and hence the relative nullity foliation $\{L\}$ on $M^{n}$ has $K_{L}=K_{\text {mix }}=1$. In particular, $\bar{M}^{n+p}$ contains a $\mu(M)$-dimensional totally geodesic submanifold $L$ (a generator from $M^{n}$ ) with sectional curvature 1 .

Lemma 9 [Bor 3]. Let $\bar{M}$ be a compact Riemannian $C^{4}$-manifold with $1 / 4<K_{\bar{M}} \leq 1$. Then any complete totally geodesic submanifold $L^{\mu}(\mu \geq 2)$ is simply connected.

In view of the curvature restrictions on $\bar{M}^{n+p}$ and by Lemma 9 the generator $L$ is simply connected and hence is isometric to the unit sphere $S^{\mu(M)}$. Hence $\bar{M}^{n+p}$ contains a closed geodesic of length $2 \pi$. Let $m_{1}, m_{2}$, $m_{3}$ be points on this geodesic which define a triangle with sides of length 
$\frac{2}{3} \pi$ each. Since $\bar{M}^{n+p}$ is simply connected with certain curvature conditions, every geodesic of length $\frac{2}{3} \pi$ is shortest (see [Top]).

TheOREM 9 [Top]. Let $\bar{M}$ be a compact Riemannian $C^{4}$-manifold with $K_{\bar{M}} \geq 1$. If there exists a triangle in $\bar{M}$ with perimeter $2 \pi$ whose sides are shortest geodesics, then $\bar{M}$ is isometric to the unit sphere.

Then we apply Toponogov's Theorem 9 to this triangle with vertices $m_{1}$, $m_{2}$ and $m_{3}$ and conclude that $\bar{M}^{n+p}$ is isometric to the unit sphere.

Theorems 7 and 8 directly yield

THEOREM 10. Let $M^{n}$ be a compact curvature invariant submanifold in a complete simply connected Riemannian space $\bar{M}^{n+p}$ with $9 / 4 \geq K \bar{M} \geq 1$ and suppose that one of the following conditions is satisfied:

1. $\operatorname{Ric}^{s}(M) \leq s$ for some $s<n-1$, and $2 p<n-\nu(n)-s+\delta_{1 s}-1$,

2. $K_{M} \leq 1, \operatorname{inj}(M) \geq \pi$ and $2 p<n-1$.

Then $\bar{M}^{n+p}$ is isometric to the unit sphere and $M^{n}$ is a totally geodesic submanifold.

Proof. 1 . Since $2 p<n-s+\delta_{1 s}-1$, by Theorem 8 the space $\bar{M}^{n+p}$ is isometric to the unit sphere $S^{n+p}$. Now $\operatorname{Ric}^{s}(\bar{M})=s$ and hence conditions 1 of Theorem 7 hold. Thus $M^{n}$ is a great sphere.

2. Since conditions 1 of Theorem 8 are satisfied, $\bar{M}^{n+p}$ is isometric to the unit sphere $S^{n+p}$ and $K_{\bar{M}}=1$. From Theorem 7, case 2, we find that $M^{n}$ is a great sphere.

Acknowledgements. I would like to thank Dr. Robert Wolak and Jerzy Trzeciak, copy editor, for their help in preparing this paper.

\section{References}

[Abe 1] K. Abe, A characterization of totally geodesic submanifolds in $S^{N}$ and $C P^{N}$ by an inequality, Tôhoku Math. J. 23 (1971), 219-244.

[Abe 2] -, Applications of a Riccati type differential equation to Riemannian manifolds with totally geodesic distributions, ibid. 25 (1973), 425-444.

[Bor 1] A. Borisenko, Complete l-dimensional submanifolds of nonpositive extrinsic curvature in a Riemannian space, Mat. Sb. 104 (1977), 559-576 (in Russian).

[Bor 2] - On external geometrical properties of parabolic submanifolds and topological properties of saddle submanifolds in symmetric spaces of rank one, ibid. 116 (1981), 440-457 (in Russian).

[Bor 3] - On extremal properties of compact parabolic submanifolds in a Riemannian space, ibid. 133 (1987), 112-126 (in Russian).

[Bor 4] - , The foliations of extrinsic negative curvature in a Riemannian space, in: Conf. on Diff. Geometry and Applications, Abstracts, Brno, 1995, 5-6. 
[BRT] A. Borisenko, M. Rabelo and K. Tenenblat, On saddle submanifolds of Riemannian manifolds, in: Conf. on Diff. Geometry, Abstracts, Budapest, 1996, $24-25$.

[DR] M. Dajczer and L. Rodríguez, On isometric immersions into complex space forms, Math. Ann. 299 (1994), 223-230.

[Fer] D. Ferus, Totally geodesic foliations, Math. Ann. 188 (1970), 313-316.

[Flo] L. Florit, On submanifolds with nonpositive extrinsic curvature, ibid. 298 (1994), 187-192.

[Fra] T. Frankel, Manifolds with positive curvature, Pacific J. Math. 11 (1961), 165171.

[Gla] V. Glazyrin, Topological and metric properties of $k$-saddle submanifolds, Dokl. Akad. Nauk SSSR 233 (1977), 1028-1030 (in Russian).

[GK] S. Goldberg and S. Kobayashi, On holomorphic bisectional curvature, J. Differential Geom. 1 (1967), 225-233.

[Ish] T. Ishihara, Radii of immersed manifolds and nonexistence of immersions, Proc. Amer. Math. Soc. 78 (1980), 276-279.

[Jac] M. Jacobowitz, Isometric embedding of a compact Riemannian manifold into Euclidean space, ibid. 40 (1973), 245-246.

[KX 1] K. Kenmotsu and C. Xia, Hadamard-Frankel type theorems for manifolds with partially positive curvature, Pacific J. Math., to appear.

[KX 2] -, Intersections of minimal submanifolds in manifolds of partially positive curvature, Kodai Math. J. 18 (1995), 242-249.

[KN] S. Kobayashi and K. Nomizu, Foundations of Differential Geometry, Vols. 1, 2, Interscience Publ., 1963, 1969.

[Mal] R. Maltz, The nullity spaces of curvature-like tensors, J. Differential Geom. 7 (1972), 519-525.

[Moo 1] J. Moore, An application of second variation to submanifold theory, Duke Math. J. 42 (1975), 191-193.

[Moo 2] -, Submanifolds of constant positive curvature, I, ibid. 44 (1977), 449-484.

[Mor] J. Morvan, Distance of two submanifolds of a manifold with positive curvature, Rend. Mat. 3 (1983), 357-366.

[O'N] B. O'Neill, Immersion of manifolds of nonpositive curvature, Proc. Amer. Math. Soc. 11 (1960), 132-134.

[Ots] T. Otsuki, On the existence of solutions of a system of quadratic equations and its geometrical application, Proc. Japan Acad. 29 (1953), 99-100.

[Rov] V. Rovenskiǔ, Submanifolds and foliations with restrictions on partial Ricci curvature, in: Problems of Mathematical Analysis, Krasnoyarsk Technical Univ., 1996, 53-62 (in Russian).

[Shef] S. Shefel', On two classes of $k$-dimensional submanifolds in $n$-dimensional Euclidean space, Sibirsk. Mat. Zh. 10 (1969), 459-467 (in Russian).

[Shen] Z. Shen, On complete manifolds of nonnegative kth-Ricci curvature, Trans. Amer. Math. Soc. 338 (1993), 289-310.

[Tan] S. Tanno, Totally geodesic foliations with compact leaves, Hokkaido Math. J. 1 (1972), 7-11.

[Top] V. Toponogov, Extremal theorems for Riemannian spaces with curvature bounded above. I, Sibirsk. Mat. Zh. 15 (1974), 1348-1371 (in Russian). 
[Wu] H. Wu, Manifolds of partially positive curvature, Indiana Univ. Math. J. 36 (1987), 525-548.

Geometry Chair

State Pedagogical University

Lebedeva st., 89

Krasnoyarsk 660049, Russia

E-mail: roven@edk.krasnoyarsk.su

Reçu par la Rédaction le 28.11.1996 